\title{
MINIMAL TUBES OF FINITE INTEGRAL CURVATURE ${ }^{\dagger}$ )
}

\section{G. Tkachëv}

UDC 517.53

Introduction. Let $x=\left(x_{1}, \ldots, x_{n}, x_{n+1}\right)$ be a point in the Euclidean space $\mathbb{R}^{n+1}$ and let $M$ be some oriented noncompact $p$-dimensional Riemannian manifold, $2 \leq p \leq n$.

Definition 1. A surface $\mathcal{M}=(M, \mathbf{u})$ given by a $C^{2}$-immersion $\mathbf{u}: M \rightarrow \mathbb{R}^{n+1}$ is called a tube with existence interval $\tau(\mathcal{M}) \subset O x_{n+1}$ if

(i) for every $\tau \in \tau(\mathcal{M})$ the sections $\Sigma_{\tau}=\mathrm{u}(\mathcal{M}) \cap \Pi_{\tau}$ by the hyperplanes $\Pi_{\tau}=\left\{x \in \mathbb{R}_{1}^{n+1}\right.$ : $\left.x_{n+1}=\tau\right\}$ are nonempty compact sets;

(ii) for arbitrary $\tau^{\prime}, \tau^{\prime \prime} \in \tau(\mathcal{M})$ the portion of $\mathcal{M}$ between two different hyperplanes $\Pi_{\tau^{\prime}}$ and $\Pi_{\tau^{\prime \prime}}$ is a compact set.

The length of the interval $\tau(\mathcal{M})$ is referred to as the existence time of the tube. where.

Definition 2. A surface $\mathcal{M}$ is said to be minimal if the mean curvature of $\mathcal{M}$ vanishes every-

Observe that the definition of tube imposes no topological constraints on the surface. On the other hand, studying the classical Plateau problem for contours with two components lying in parallel planes leads naturally to the class of tubular surfaces. Minimal two-dimensional tubes were first considered in Nitsche's article [1]. Nitsche also proved that the existence time of a tube can be bounded above using the sizes of boundary components. On use made of various versions of the geometric maximum principle, in [2] this inequality was improved and translated to the multidimensional case (see also $[3,4])$.

It is well known that in the multidimensional case $(\operatorname{dim} M \geq 3)$ every minimal tube of arbitrary codimension has finite existence time $|\tau(\mathcal{M})|[2,5,6]$. In this case the existence time of a tube is bounded above using the minimal girth radius of the surface, the diameter of the minimal section $\Sigma_{\tau}$.

In this respect, the two-dimensional case is specific. Namely, as it is seen from the examples of the standard catenoid and the one-dimensional family of Riemann surfaces of [7] (the so-called "skew catenoids"), the existence time $|\tau(\mathcal{M})|$ of minimal two-dimensional tubes may be infinite as well as finite (inextensible). Moreover, in the latter case a Riemann surface may have an arbitrary prescribed finite value $|\tau(\mathcal{M})|$, provided that the minimal girth radius is fixed. This circumstance dictates the necessity of introducing extra characteristics in whose terms we could estimate the existence time. In the present article, we discuss the following question: under what conditions can we guarantee that of the existence time of a tube is finite?

In this regard, the author has announced a new approach [8] which applies to doubly connected tubes with single-valued Gaussian map and which is based on the methods of the conformal modulus of families of curves on a surface. In the present article, we extend this approach to minimal tubes of arbitrary topological structure with finite total curvature. The last requirement is necessary. We show this by examples at the end of the article. The flux vector of a minimal tube plays the role of an extra characteristic. It is an invariant of the tube under the semigroup of motions preserving the time axis. The corresponding definition is as follows:

Let $e^{\top} \equiv e^{\top}(m)$ denote the orthogonal projection of a vector $e \in \mathbb{R}^{n+1}$ onto the tangent space of a surface $\mathcal{M}$ at a point $m$ and let $\tau \in \tau(\mathcal{M})$ be a regular value of the coordinate function $u_{n+1}(m)$ (i.e., $\left.e_{n+1}^{\top} \neq 0\right)$. Then $\Sigma_{\tau}$ is a finite union of compact $(p-1)$-dimensional submanifolds without boundary and $\nu=e_{n+1}^{\top} /\left|e_{n+1}^{\top}\right|$ is a continuous field of unit normals to $\Sigma_{\tau}$ with respect to $\mathcal{M}$ which are oriented in the direction $e_{n+1}$.

†) The research was financially supported by St. Petersburg University (Grant 95-0-1.9-34).

Volgograd. Translated from Sibirskǐ Matematicheski⿱ Zhurnal, Vol. 39, No. 1, pp. 181-190, January-February, 1998. Original article submitted February 16, 1996. 
Definition 3. The vector $J(\mathcal{M}) \in \mathbb{R}^{n+1}$ with coordinates

$$
J_{k}=\int_{\Sigma_{\tau}}\left\langle e_{k}^{\top} ; \nu\right\rangle, \quad 1 \leq k \leq n+1,
$$

is called the flux vector of the minimal tube $\mathcal{M}$.

Observe that, by harmonicity of the coordinate functions [9], the vector $J(\mathcal{M})$ is independent of the choice of $\tau \in \tau(\mathcal{M})$ and, owing to the orientation of $\nu$, the $(n+1)$ th coordinate of $J(\mathcal{M})$ is strictly positive. Denote by $\alpha(\mathcal{M})$ the angle between $J(\mathcal{M})$ and the axis $O x_{n+1}$. As follows from the definition, the length of the flux vector and the angle $\alpha(\mathcal{M})$ are invariant under the semigroup of motions of $\mathbb{R}^{n+1}$ preserving the direction of the time axis.

It is also worth making a physical interpretation of the flux vector. Namely, the minimal tubes are Euclidean analogs of closed relativistic strings in Minkowski space [10]. In such approach, the geometric invariants of tubes represent certain physical characteristics of the corresponding elementary particles. For instance, the length of $\tau(\mathcal{M})$ corresponds to the existence time of a string and the flux vector $J(\mathcal{M})$, to its momentum [11].

The main result of the present article is the following theorem:

Theorem 1. Suppose that $\mathcal{M}$ is a minimal two-dimensional tube in $\mathbb{R}^{3}$ of an arbitrary topological type and with flux vector $J(\mathcal{M})$. Then if the absolute integral Gaussian curvature $G(\mathcal{M})$ is finite and $\alpha(\mathcal{M})>0$ then the existence time $|\tau(\mathcal{M})|$ of $\mathcal{M}$ is finite and

$$
|\tau(\mathcal{M})| \leq \frac{G(\mathcal{M})\|J(\mathcal{M})\| \cos \alpha(\mathcal{M})}{16 \alpha^{2}(\mathcal{M})} .
$$

This assertion implies that the existence time of a minimal tube with Gaussian map of finite multiplicity (in particular, single-valued) is finite, since such a surface satisfies the inequality $G(\mathcal{M}) \leq$ $4 \pi s$, where $s$ is the multiplicity of the map. Namely, the following generalization of the corresponding result of [8] holds:

Corollary 1. Suppose that $\mathcal{M}, \operatorname{dim} \mathcal{M}=2$, is a minimal tube in $\mathbb{R}^{3}$ of an arbitrary topological type. If the Gaussian map is at most $s$-valent and $\alpha(\mathcal{M})>0$ then the existence time $|\tau(\mathcal{M})|$ of $\mathcal{M}$ is finite and

$$
|\tau(\mathcal{M})| \leq \frac{\pi s\|J(\mathcal{M})\| \cos \alpha(\mathcal{M})}{4 \alpha^{2}(\mathcal{M})} .
$$

REMARK 1. The traditional characteristic, the integral Gaussian curvature, is more flexible than multiplicity in this sense. Indeed, the finiteness of the integral Gaussian curvature does not imply finiteness for the multiplicity of the Gaussian map a priori. On the other hand, the examples of tubes with an arbitrary angle $\alpha(\mathcal{M})$ and an infinite existence time given in the last part of the present article demonstrate the necessity of constraints on the Gaussian map.

In the case of tubes with an infinite existence time and a nonzero slope of the flux vector with respect to the time axis, (1) also yields a uniform linear lower estimate for the integral Gaussian curvature of an arbitrary portion of the tube which lies in a parallel layer in terms of the width of the layer.

REMARK 2. It is worthy to observe that, unlike most articles concerning minimal surfaces of finite integral curvature, we do not presume intrinsic completeness of the surface. Moreover, as follows from the results of [12], a two-dimeisional tube with finite existence time and finite integral curvature cannot be a complete surface (with respect to the intrinsic metric).

1. A Weierstrass-type representation for doubly connected tubes. In the current section, we suppose that $\mathcal{M}$ is a two-dimensional doubly connected minimal tube in $\mathbb{R}^{3}$ given as an immersion of some ring. Then the results by Osserman [13] and Schiffman [14] imply existence of a global system of isothermal coordinates on $M$. Moreover, $\mathcal{M}$ may be assumed to be conformally equivalent to the ring $D_{R}=\{z: 1 / R<|z|<R\}$ for a suitable $R>1$. 
Let $g(\zeta)$ be a holomorphic function in the ring $D_{R}$, let $C_{t}=\{z \in \mathbb{C}:|z|=t\}$, and let

$$
a_{0}(g)=\frac{1}{2 \pi i} \int_{C_{1}} \frac{g(\zeta) d \zeta}{\zeta}
$$

be the central coefficient of the Laurent expansion of $g(\zeta)$.

Definitron 4. We say that $g(\zeta)$ is admissible for $D_{R}$ if it is nonvanishing and

$$
a_{0}(g)=-\overline{a_{0}(1 / g(z))} .
$$

The following Weierstrass-type representation holds:

Lemma 1. Under the above assumptions, the surface $\mathcal{M}$ admits the parameterization

$$
\mathbf{u}(z)=\operatorname{Re} \int_{z_{0}}^{z} \mathbf{F}(\zeta) d \zeta
$$

where

$$
\mathbf{F}=\left(F_{1} ; F_{2} ; F_{3}\right)=\frac{J_{3}}{4 \pi}\left(\frac{1-g^{2}(z)}{g(z) z} ; \frac{i\left(1+g^{2}(z)\right)}{g(z) z} ; \frac{2}{z}\right)
$$

and $g(z)$ is some holomorphic function admissible for $D_{R}$ and such that

$$
a_{0}(g)=-\frac{1}{J_{3}}\left(J_{1}+i J_{2}\right), \quad a_{0}(1 / g)=\frac{1}{J_{3}}\left(J_{1}-i J_{2}\right),
$$

with $J_{k}$ the components of the flux vector of $\mathcal{M}$.

Proof. In line with [13], we write down the classical Weierstrass-Enneper parameterization of $\mathcal{M}$. Namely, there are holomorphic functions $f(z)$ and $g(z)$ in $D_{R}$ such that (4) is valid for the holomorphic vector-function

$$
\mathbf{F}(z)=\left(\left(1-g^{2}(z)\right) f(z) ; i\left(1+g^{2}(z)\right) f(z) ; 2 f(z) g(z)\right)
$$

and

$$
\operatorname{Re} \int_{C_{1}} \mathbf{F}(\zeta) d \zeta=0
$$

Moreover, $g(z)$ is the composition of the Gaussian map of $\mathcal{M}$ followed by the stereographic projection of the unit sphere, with respect to the north pole, to the tangent plane at the south pole.

First observe that, with the above notations,

$$
J(\mathcal{M})=\operatorname{Im} \int_{|z|=1} F(\zeta) d \zeta .
$$

Indeed, consider the conjugate functions

$$
v_{k}(z)=\operatorname{Im} \int_{z_{0}}^{z} F_{k}(\zeta) d \zeta
$$


In general, $v_{k}(z)$ is a many-valued harmonic function. On the other hand, the gradient $\nabla v_{k}$ is well defined. Using the properties of the Hodge *-operator, we obtain

$$
\begin{gathered}
J_{k}(\mathcal{M})=\int_{\Sigma_{\tau}}\left\langle\nabla u_{k} ; \nu\right\rangle d s=\int_{\Sigma_{\tau}}\left\langle * \nabla u_{k} ; * \nu\right\rangle d s \\
=\int_{\Sigma_{\tau}}\left\langle\nabla v_{k} ; * \nu\right\rangle d s=\int_{\Sigma_{\tau}} d v_{k}=\operatorname{Im} \int_{|z|=1} F_{k}(\zeta) d \zeta
\end{gathered}
$$

and thereby (8) is proven.

By (4), the coordinate function $u_{3}(z)$ is harmonic in $D_{R}$. Using the definition of tube, we obtain

$$
\lim _{z \rightarrow 1 / R} u_{3}(z)=\tau_{1}, \quad \lim _{z \rightarrow R} u_{3}(z)=\tau_{2},
$$

where $\tau(\mathcal{M})=\left(\tau_{1} ; \tau_{2}\right)$ is the interval of the projection of $\mathcal{M}$ to the $O x_{3}$-axis.

Consider the auxiliary harmonic function

$$
h(z)=\tau_{1}+\frac{\tau_{2}-\tau_{1}}{2 \log R} \log (R|z|) .
$$

It is easy to see that $h(z)$ as well satisfies (9) and, thus, $h_{1}(z)=u_{3}(z)-h(z)$ is a harmonic function in the ring which meets the boundary condition

$$
\lim _{z \rightarrow \partial D_{R}} h_{1}(z)=0 .
$$

Applying the maximum principle, we obtain $h_{1}(z) \equiv 0$ everywhere in $D_{R}$; hence,

$$
u_{3}(z) \equiv \tau_{1}+\frac{\tau_{2}-\tau_{1}}{2 \log R} \log (R|z|) .
$$

In particular, (10) implies that the differential $d u_{3}(z)$ vanishes nowhere in $D_{R}$. Consequently, the normal $n(z)$ to $\mathcal{M}$ is parallel to $e_{3}$ at no point. Recalling the geometric interpretation of $g(z)$, we infer that $g(z): D_{R} \rightarrow \mathbb{C} \backslash\{0 ; \infty\}$.

Comparing (10) and (6), we conclude that

$$
2 g(z) f(z)=\frac{\tau_{2}-\tau_{1}}{2 \log R} \cdot \frac{1}{z} .
$$

To exclude $\log R$ from the last equality, we insert (11) in (6). Using (8), we obtain

$$
\log R=\frac{\pi\left(\tau_{2}-\tau_{1}\right)}{J_{3}} .
$$

Finally, substituting the so-obtained relation in (11), we arrive at (4). Thus,

To prove (5), we write down the condition (8) by using the above relation between $f(\zeta)$ and $g(\zeta)$.

$$
\int_{C_{1}} \frac{1-g^{2}(\zeta)}{2 g(\zeta)} \frac{d \zeta}{\zeta}=\frac{2 \pi J_{1} i}{J_{3}}, \quad \int_{C_{1}} \frac{1+g^{2}(\zeta)}{2 g(\zeta)} \frac{d \zeta}{\zeta}=\frac{2 \pi J_{2}}{J_{3}},
$$

whence, after a simplification, we obtain the sought identity. The lemma is proven. 
2. An estimate for the existence time for doubly connected tubes.

DEFINITION 5. Suppose that $\Gamma$ is some family of locally rectifiable curves $\gamma \subset D$ on the complex plane and $\varphi(z) \geq 0$ is a nonnegative Borel function such that

$$
\int_{\gamma} \varphi(z)|d z| \geq 1
$$

for all $\gamma \in \Gamma$. The infimum

$$
\bmod \Gamma=\inf \iint_{D} \varphi^{2}(z) d z d \bar{z}
$$

over all such $\varphi(z)$ is called the conformal modulus of $\Gamma$.

Let $\Gamma(R)$ be the family of concentric circles $C_{t}, t \in(1 / R ; R)$, with center the origin and radius $t$, which are included in the ring $D_{R}$. It is well known (see, for instance, $[15$, p. 19]) that

$$
\bmod \Gamma(R)=\frac{\log R}{\pi}
$$

Below, we also need the following classical formulas for some intrinsic characteristics of a minimal surface parameterized by (6). Namely, the line element of $\mathcal{M}$ and the Gaussian curvature are as follows (see [13]):

$$
\begin{gathered}
d s \equiv \lambda(z)|d z|=|f|\left(1+g^{2}\right)|d z|, \\
K=-\left(\frac{2\left|g^{\prime}\right|}{|f|\left(1+|g|^{2}\right)^{2}}\right)^{2} .
\end{gathered}
$$

Using the fact that the coordinates are isothermal and Lemma 1, we obtain the following expression for the absolute integral Gaussian curvature $G(\mathcal{M})$ :

$$
G(\mathcal{M}) \equiv-\iint_{D_{R}} K(z) \lambda^{2}(z) d z d \bar{z}=\iint_{D_{R}} \frac{4\left|g^{\prime}\right|^{2}}{\left(1+|g|^{2}\right)^{2}} d z d \bar{z}
$$

Lemma 2. The estimate (1) is valid for every doubly connected minimal tube $\mathcal{M}$ with finite absolute Gaussian curvature $G(\mathcal{M})$.

Proof. Let $g(\zeta)$ be the function of Lemma 1 corresponding to $\mathcal{M}$ and let $a_{0}(g)=a e^{i \theta}$ be the central Laurent coefficient of $g$ in polar form for some positive real $a$. Then from (5) we obtain $a_{0}(1 / g)=-a e^{-i \theta}$. Consequently, the modified function $g_{1}(\zeta)=e^{-i \theta} g(\zeta)$ satisfies the following equalities for every $t \in(1 / R ; R)$ :

$$
a_{0}\left(g_{1}\right)=\frac{1}{2 \pi} \int_{0}^{2 \pi} g_{1}\left(t e^{i \xi}\right) d \xi=a, \quad a_{0}\left(1 / g_{1}\right)=\frac{1}{2 \pi} \int_{0}^{2 \pi} \frac{d \xi}{g_{1}\left(t e^{i \xi}\right)}=-a
$$

Put

$$
\begin{aligned}
\varphi(\zeta) & \equiv \frac{\left|g_{1}^{\prime}(\zeta)\right|}{1+\left|g_{1}(\zeta)\right|^{2}}=\frac{\left|g^{\prime}(\zeta)\right|}{1+|g(\zeta)|^{2}} \\
\sigma & =\inf _{1 / R<t<R} \int_{C_{t}} \varphi(\zeta)|d \zeta| .
\end{aligned}
$$


By Definition 5, we have the following estimate for the value of the conformal modulus $\bmod \Gamma(R)$ :

$$
\bmod \Gamma(R) \leq \frac{1}{\sigma^{2}} \iint_{D_{R}} \varphi^{2}(z) d z d \bar{z} .
$$

Recalling (12) and (13), whence we obtain

$$
\log R \leq \frac{\pi G(\mathcal{M})}{4 \sigma^{2}} .
$$

Now, we estimate $\sigma$ from below. To this end, consider the auxiliary stereographic mapping

$$
h(\zeta)=\left(\frac{2 \operatorname{Re} \zeta}{|\zeta|^{2}+1} ; \frac{2 \operatorname{Im} \zeta}{|\zeta|^{2}+1} ; \frac{|\zeta|^{2}-1}{|\zeta|^{2}+1}\right): \mathbb{C}^{1} \rightarrow S^{2} \backslash\{P\}
$$

of the complex plane onto the unit sphere $S^{2}$ without the north pole $P=(0 ; 0 ; 1)$. The differential of the stereographic mapping at a point $w$ is calculated by the formula

$$
\left|d_{w} h\right|=\frac{2|d w|}{|w|^{2}+1} .
$$

Thereby for every $t \in(1 / R ; R)$ we have

$$
\begin{gathered}
\int_{C_{t}} \varphi(\zeta)|d \zeta| \equiv \int_{C_{t}} \frac{\left|g_{1}^{\prime}(\zeta)\right|}{1+\left|g_{1}(\zeta)\right|^{2}}|d \zeta|=\int_{g_{1}\left(C_{t}\right)} \frac{|d w|}{1+|w|^{2}} \\
=\frac{1}{2} \int_{h \circ g_{1}\left(C_{t}\right)}|d h| \geq \frac{1}{2} \ell\left(h \circ g_{1}\left(C_{t}\right)\right),
\end{gathered}
$$

where $\ell(E)$ is the length of a continuum $E \in S^{2}$ in the spherical metric.

Combining (15) and (17), we obtain

$$
\sigma \geq \frac{1}{2} \inf _{1 / R<t<R} \ell\left(h \circ g_{1}\left(C_{t}\right)\right)
$$

Taking real parts in (14) and applying the mean value theorem, we conclude that for every $t \in(1 / R ; R)$ there are $\xi_{1}$ and $\xi_{2}$ such that

$$
\operatorname{Re} g_{1}\left(t e^{i \xi_{1}}\right)=a, \quad \operatorname{Re} \frac{1}{g_{1}\left(t e^{i \xi_{2}}\right)}=-a .
$$

Then it follows from (19) that $\gamma_{t}=g_{1}\left(C_{t}\right)$ has a nonempty intersection with the straight line $L_{1}=$ $\{z \in \mathbb{C}: \operatorname{Re} z=a\}$ and the circle $L_{2}=\left\{z \in \mathbb{C}: \operatorname{Re}\left(\frac{1}{z}\right)=-a\right\}$. Recalling that $\gamma_{t}$ is a closed curve and using the equality $h \circ g_{1}\left(C_{t}\right)=h\left(\gamma_{t}\right)$, from (18) we derive

$$
\sigma \geq \frac{1}{2} \cdot 2 \operatorname{dist}\left(h\left(L_{1}\right) ; h\left(L_{2}\right)\right)=\operatorname{dist}\left(h\left(L_{1}\right) ; h\left(L_{2}\right)\right),
$$

where dist stands for the geodesic distance between the corresponding subsets of the unit sphere.

Consider an arbitrary point $h(z)=\left(x_{1}, x_{2}, x_{3}\right) \in h\left(L_{1}\right)$. Using the definition of the stereographic projection and the fact that $\operatorname{Re} z=a$ on $L_{1}$, we obtain

$$
x_{1}+a x_{3}=\frac{2 \operatorname{Re} z}{1+|z|^{2}}+a \frac{|z|^{2}-1}{|z|^{2}+1}=a .
$$


This means that $h\left(L_{1}\right)$ is a circle on $S^{2}$ lying in the plane $x_{1}+a x_{3}=a$. Similarly, we verify that $h\left(L_{2}\right)$ is the circle, in the plane $x_{1}+a x_{3}=-a$, which is centrally symmetric to $h\left(L_{1}\right)$. Direct calculation of the distance between these circles with respect to the metric of the unit sphere yields

$$
\operatorname{dist}\left(h\left(L_{1}\right) ; h\left(L_{2}\right)\right)=2 \tan ^{-1} a .
$$

Involving (16) and (20), we now obtain

$$
\log R \leq \frac{\pi G(\mathcal{M})}{16\left(\tan ^{-1}\right)^{2} a} .
$$

Suppose that $\alpha(\mathcal{M})$ is the angle between the flux vector $J(\mathcal{M})$ and the $O x_{3}$-axis. Then

$$
\tan \alpha(\mathcal{M})=\frac{\sqrt{J_{1}^{2}+J_{2}^{2}}}{J_{3}}=a .
$$

Using (21), we arrive at the sought inequality. The lemma is proven completely.

3. Proof of Theorem 1. Let $\mathcal{M} \subset \mathbb{R}^{3}$ be a two-dimensional immersed minimal tube of an arbitrary topological type with the existence interval $\tau(\mathcal{M})=\left(\tau_{1} ; \tau_{2}\right)$. Denote by $E$ the set of all critical points of the coordinate function $u_{3}(\zeta)$ of the immersion $\mathbf{u}: M \rightarrow \mathbb{R}^{3}$. Observe that, by harmonicity of $u_{3}(\zeta), E$ comprises only isolated points; in particular, $E$ is at most countable. Fix $\varepsilon>0$. Then, by Definition 1, the set $\left\{h_{i}=u_{3}\left(\xi_{i}\right): \xi_{i} \in E\right\}$ of the critical values in the interval $\left(\tau_{1}+\varepsilon ; \tau_{2}-\varepsilon\right)$ is at most finite. This set may be assumed to be nonempty for a sufficiently small $\varepsilon>0$ : otherwise the surface would be an immersed ring and the claim of the theorem would ensue from Lemma 2. Moreover, without loss of generality we may assume that $h_{i}, 1 \leq i \leq N$, are enumerated in increasing order.

Fix an arbitrary index $i: 1 \leq i \leq N-1$. Then the portion of $\mathcal{M}$ in the space layer $h_{i} \leq x_{3} \leq h_{i+1}$ splits into finitely many connected minimal tubes $D_{1}, \ldots, D_{k}$ each having the topological type of a ring. Indeed, by the classical Morse lemma, the absence of critical points of the height function $u_{3}$ on every component $D_{j}$ implies that $D_{j}$ is homeomorphic to an (immersed) cylinder.

Denote by $J^{(j)}=J\left(D_{j}\right)$ the flux vector that corresponds to the component $D_{j}$ regarded as an individual tube and denote by $\alpha_{j}$ the angle between $J^{(j)}$ and the basis vector $e_{3}$. Then, for every $j, 1 \leq j \leq k$, from Lemma 2 we obtain

$$
h_{i+1}-h_{i} \leq \frac{G\left(D_{j}\right) J_{3}\left(D_{j}\right)}{16 \alpha_{j}^{2}} \leq \frac{G(D) J_{3}\left(D_{j}\right)}{16 \alpha_{j}^{2}}
$$

where $D=\cup D_{j}$. Moreover, by the definition of flux vector, we have

$$
J^{(1)}+J^{(2)}+\ldots+J^{(k)}=J(\mathcal{M}) .
$$

Recalling that the third coordinate

$$
J_{3}^{(i)} \leq \sum_{j=1}^{k} J_{3}^{(j)}=J_{3}(\mathcal{M})
$$

of the flux vector is nonnegative, we obtain

$$
h_{i+1}-h_{i} \leq \frac{G(D) J_{3}(\mathcal{M})}{16 \alpha_{j}^{2}} .
$$


On the other hand, there is an index $\nu$ such that $\alpha_{\nu} \geq \alpha(\mathcal{M})$. Indeed, consider the convex polyhedron $P$ that is the convex hull of the origin $O \in \mathbb{R}^{3}$ and the ends of the vectors $J^{(j)}$. Using (23), we infer that the vector $\frac{1}{k} J(\mathcal{M})$ lies in $P$. Then the convexity of $P$ implies that there is a vector $J^{(\nu)}$ such that the angle $\alpha_{\nu}$ is not less than the angle between $e_{3}$ and $\frac{1}{k} J(\mathcal{M})$, the latter coincident with $\alpha(\mathcal{M})$ by definition. Thereby, applying (24) to $\nu$, we obtain

$$
h_{i+1}-h_{i} \leq \frac{G(D) J_{3}(\mathcal{M})}{16 \alpha^{2}(\mathcal{M})} .
$$

Summing the so-obtained inequalities over all $i$, we arrive at the estimate

$$
|\tau(\mathcal{M})|-2 \varepsilon \leq \frac{G\left(\mathcal{M}^{\prime}\right) J_{3}(\mathcal{M})}{16 \alpha^{2}(\mathcal{M})}
$$

where $\mathcal{M}^{\prime}$ is the part of $\mathcal{M}$ in the layer $\tau_{1}+\varepsilon \leq x_{3} \leq \tau_{2}-\varepsilon$. Recalling that $\varepsilon$ is arbitrary and that the absolute integral Gaussian curvature, considered as a set function, increases, we arrive at the sought inequality. The theorem is proven completely.

4. Tubes with infinite existence time. In this section, we give examples which show that there are minimal tubes whose existence interval is $\mathbb{R}$ and the slope of the flux vector is arbitrary.

Lemma 3. Let $\psi(\zeta)$ be a holomorphic function in $D_{R}$ such that

$$
\overline{\psi\left(-\frac{1}{z}\right)}=-\psi(z)
$$

Then, for every integer $N$, the function

$$
g(z)=z^{2 N+1} \exp (\psi(z))
$$

is admissible for $D_{R}$.

Proof. Put $w=-1 / z$. Then

$$
\begin{aligned}
\frac{1}{g(z)} & =z^{-2 N-1} \exp (-\psi(z))=-\left(-\frac{1}{z}\right)^{2 N+1} \cdot \exp \overline{\psi\left(-\frac{1}{z}\right)} \\
& =-w^{2 N+1} \overline{\exp \psi(\bar{w})}=-\overline{\bar{w}^{2 N+1} \exp \psi(\bar{w})}=-\overline{g(\bar{w})}
\end{aligned}
$$

and, consequently, for the central Laurent coefficients we have

$$
a_{0}(1 / g(z))=a_{0}(-\overline{g(\bar{w})})=-\overline{a_{0}(g(w))}=-\overline{a_{0}(g(z))},
$$

as required.

As an application, we consider the simplest function satisfying (25):

$$
\psi(z)=\lambda\left(z+\frac{1}{z}\right)
$$

where $\lambda \geq 0$ is some positive real. By Lemma 3 , the function

$$
g_{\lambda}(z)=z \exp \lambda\left(z+\frac{1}{z}\right)
$$


is admissible for the punctured complex plane $D_{\infty} \equiv \mathbb{C} \backslash\{0\}$. Taking the Laurent expansion of this function, we find the following expression for the central Laurent coefficient of $g_{\lambda}(z)$ :

$$
a_{0}\left(g_{\lambda}\right)=\sum_{k=1}^{\infty} \frac{\lambda^{2 k-1}}{k !(k-1) !}
$$

Let $\mathcal{M}_{\lambda}$ be the minimal doubly connected tube given by (4) with the function $g_{\lambda}(z)$. Then $\mathcal{M}_{\lambda}$ is conformally equivalent to $\mathbb{C} \backslash\{0\}$ and, applying (20) and (22), we derive

$$
\tan \alpha\left(\mathcal{M}_{\lambda}\right)=\left|a_{0}\left(g_{\lambda}\right)\right|=\sum_{k=1}^{\infty} \frac{\lambda^{2 k-1}}{m !(m-1) !}
$$

Varying $\lambda \in(0 ;+\infty)$, we thus obtain doubly connected minimal tubes $\mathcal{M}_{\lambda}$ with infinite existence interval and an arbitrary slope of the flux vector with respect to the $O x_{3}$-axis.

\section{References}

1. J. C. C. Nitsche, "On new results in the theory of minimal surfaces," Matematika, 11, No. 3, 37-100 (1967).

2. A. D. Vedenyapin and V. M. Miklyukov, "Exterior sizes for tubular minimal hypersurfaces," Mat. Sb., 131, No. 2, 240-250 (1986).

3. Yu. D. Burago and V. A. Zalgaller, Geometric Inequalities [in Russian], Nauka, Leningrad (1986).

4. U. Dierkes, "Maximum principles and nonexistence results for minimal submanifolds," Manuscripta Math., 69, No. 2, 203-218 (1990).

5. V. M. Miklyukov and V. G. Tkachēv, "Some properties of tubular minimal hypersurfaces of arbitrary codimension," Mat. Sb., 180, No. 9, 1278-1295 (1989).

6. V. A. Klyachin, "An estimate of length for tubular minimal surfaces of arbitrary codimension," Sibirsk. Mat. Zh., 33, No. 5, 201-205 (1992).

7. B. Riemann, Qeuvres Mathematiques de Riemann, Gautheriers-Villars, Paris (1898).

8. V. G. Tkachev, "Minimal tubes and coefficients of holomorphic functions in annulus," Bull. Soc. Sci. Lett. Lódź. Sér. Rech. Déform., 20, 19-26 (1995).

9. Sh. Kobayashi and K. Nomizu, Foundations of Differential Geometry. Vol. 2 [Russian translation], Nauka, Moscow (1981).

10. A. D. Vedenyapin, A. A. Klyachin, V. A. Klyachin, V. M. Miklyukov, and V. G. Tkachëv, "Tubes and bands of zero mean curvature in Euclidean and Pseudo-Euclidean spaces, in: Abstracts: The International Conference on Non-Euclidean Geometry (August 18-20, 1992), Kazan', 1992, pp. 19-20.

11. M. B. Green, J. H. Schwarz, and E. Witten, Superstring Theory. Vol. 1: Introduction [Russian translation], Mir, Moscow (1990).

12. Y. Fang and W. H. Meeks, "Some global properties of complete minimal surfaces of finite topology in $\mathbf{R}^{3},{ }^{n}$ Topology, 30, No. 1, 9-20 (1991).

13. R. Osserman, A Survey of Minimal Surfaces, Dover Publications, New York (1987).

14. M. Schiffman, "On surfaces of stationary area bounded by two circles, or convex curves, in parallel planes," Ann. of Math., 63, 77-90 (1956).

15. L. V. Ahlfors, Lectures on Quasiconformal Mappings [Russian translation], Mir, Moscow (1969). 